\title{
Association between Asthma Medication Adherence and Patient-Provider Collaboration
}

\author{
Lauren Lockwood \\ College of Public Health and Health Professions, University of Florida
}

Faculty mentor: David Fedele, Department of Clinical and Health Psychology

\begin{abstract}
Asthma is a significant pediatric public health concern, with $8.3 \%$ of children in the U.S. diagnosed with asthma. Pediatric asthma morbidity is consistently linked to medication non-adherence. Medication adherence has been shown to be influenced by patient-provider interactions. The aim of this study is to examine the relationship between medication adherence, using both objective and subjective reports, to patient-provider collaboration in adolescents with poorly controlled asthma. Thirty adolescents ages 12 to 15 were interviewed along with their caregivers using the Family Asthma Management System Scale (FAMSS). Adherence data were collected via pharmacy refill reports and scores on the FAMSS adherence rating subscale. Patient-provider collaboration was assessed using the corresponding subscale from the FAMSS interview. Pearson correlations were conducted to examine the relationships between both measures of adherence and patient-provider collaboration. There was no significant association between adherence measured with pharmacy refills and patient-provider collaboration $(r=.27, p=.176)$. A significant correlation was found between medication adherence, as measured by the FAMSS ratings, and patient-provider collaboration $(r=.53, p=.003)$. These findings suggest that more collaborative patient-provider relationships may be linked to higher medication adherence among adolescents with poorly controlled asthma.
\end{abstract}

\section{Introduction}

Asthma is one of the most prevalent chronic diseases affecting children in the United States. Recent data show approximately $8.3 \%$ of children are affected by asthma (Akinbami, Simon \& Rossen, 2015). The etiology of asthma is known to be impacted by genetic and environmental factors, but the exact causes of asthma and explanations of asthma severity are unknown (Zahran, Bailey, Qin \& Moorman, 2014). Asthma is a unique chronic illness in that the signs and symptoms change over time and present themselves differently in each individual case (Taylor et al., 2008). Among all children affected by this chronic disease, over $60 \%$ of cases are characterized as persistent asthma (Zahran, Bailey, Qin \& Moorman, 2014). 
Adolescents ages 12-15 have the highest number of near-fatal asthma episodes (Randolph \& Fraser, 1999). The severe nature of these episodes is often the result of failure to acknowledge or recognize the worsening of symptoms (Randolph \& Fraser, 1999). This could be attributed to the developmental changes adolescents undergo that affect their asthma management skills. Some of these transitions in development include increased autonomy, cognitive growth, knowledge gained through life experiences, and realization of identity (Bruzzese et al. 2004).

Inhaled corticosteroids (ICS) are an effective and frequently prescribed medication for poorly controlled asthma as detailed by the National Asthma Education and Prevention Program (NAEPP, 2007). ICS reduce inflammation in the lungs, thus preventing asthma exacerbations when used regularly. They also maximize the effects of bronchodilators prescribed for emergency use during asthma exacerbations. Despite the host of benefits regular ICS use offers in asthma management, adherence is typically poor in pediatric cases (McQuaid, Kopel, Klein \& Fritz, 2003). Pediatric asthma medication adherence estimates range from 46 to $58 \%$ across the literature (Modi \& Quittner, 2006; Morton, Everard \& Elphick, 2014; Walders, Kopel, KoinisMitchell \& McQuaid, 2005). Factors that contribute to non-adherence include the demanding nature of the treatment regimen, deciding when to take emergency medications, and necessity of taking medication despite lack of symptoms (Drotar \& Bonner, 2009). The adolescents' desire for increased autonomy in disease management, along with the redistribution of responsibilities within the family, can also lead to non-adherence (Walders, Drotar \& Kercsmar, 2000). Nonadherence contributes to high rates of pediatric asthma morbidity (Drotar \& Bonner, 2009), along with increased rates of hospitalizations, decreased pulmonary function, and more activity restrictions (Bauman et al. 2002).

Presently, there are no standardized measures for assessing medication adherence, making it difficult to quantify non-adherence in clinical applications. Some common approaches include subjective measures of adherence from patient, family member, and physician reports; objective measures of adherence from pill counts, pharmacy refill records, or electronic medication monitoring systems; and biochemical measurements (Brown \& Bussell, 2011). An ideal approach combines multiple methods of adherence since significant differences are typically found between estimates of adherence obtained subjectively and objectively (Krishnan et al., 2012). Therefore, there is a need to develop a combined approach in order to effectively measure medication adherence. 
The national guidelines for asthma diagnosis and management have indicated that positive relationships among patients, caregivers, and providers are necessary in disease management. A common perceived barrier for adolescent asthma self-management includes negative feelings towards providers (Rhee, Belvea, Ciurzynski \& Brasch, 2009). The provider should welcome patient questions and input to develop an asthma management plan that the patient will comply with (Jones, 2008). In a study assessing the barriers to treatment adherence in adolescents with asthma and cystic fibrosis, significant disparities were found among prescribed treatment plans and the caregiver understanding of the plan. Only $31 \%$ of providers and caregivers agreed on the asthma medications prescribed, likely indicating poor patient-provider communication (Modi \& Quittner, 2006).

Without a collaborative relationship between the patient and the physician, adherence will suffer (Sleath et al., 2012). Despite the established relationship between medication adherence and patient-provider interaction, it remains unclear whether subjective or objective measures of adherence exhibit a stronger correlation to adolescents' relationships with their providers. The goal of this paper is to examine the association between asthma medication adherence, using objective and subjective measures, and patient-provider collaboration in adolescents with poorly controlled asthma.

\section{Aims and Hypotheses}

- Aim 1: Examine the association between subjective assessment of asthma medication adherence and patient-provider collaboration.

○ Hypothesis 1: Higher asthma medication adherence based on the Family Asthma Management System Scale (FAMSS) subscale rating scores will be associated with better patient-provider collaboration.

- Aim 2: Examine the association between objective assessment of medication adherence using pharmacy refill data and patient-provider collaboration.

○ Hypothesis 2: More frequent asthma medication refill will be associated with better patient-provider collaboration.

\section{Methods}

This study looked at the association between adherence with asthma medications and the patient-provider relationship in early adolescents. Data came from baseline data collected 
through an ongoing longitudinal study titled AIM2ACT: Applying Interactive Mobile health to Asthma Care in Teens (Fedele et al. 2018). Pharmacy refill data was obtained by phoning the participants' pharmacies and recording the number of times their inhaled corticosteroids (ICS) were filled over the 12-month period prior to enrollment in the study. The adolescents included in the study were ages $12-15$ and lived in the same residence as their caregiver. All participants had a diagnosis of persistent asthma and were prescribed a controller medication. Every adolescent had poorly controlled asthma which was defined by the NAEPP as experiencing any one of the following in the past two weeks: more than 4 days of daytime asthma symptoms, 1 or more nights of nighttime awakenings, more than 4 days of normal activity limitation, more than 4 days of using short-acting beta antagonist (i.e., rescue inhaler), or a score of $\leq 19$ on the Asthma Control Test (NAEPP, 2007; Liu et al., 2007). All dyads spoke English. Those families that were enrolled in an asthma management intervention or had a child with a severe cognitive or developmental delay were excluded from the study.

The aims of AIM2ACT were twofold: to develop a mHealth tool to guide helpful caregiver support during the development of asthma self-management behaviors in early adolescents and to determine the feasibility, acceptability and efficacy of the mHealth tool among 50 early adolescents with persistent asthma.

The Family Asthma Management System Scale (FAMSS) is a semi-structured, validated family interview that was administered to the adolescents and caregivers in this study (McQuaid, Walders, Kopel, Fritz \& Klinnert, 2005). The questions in the interview required both openended responses and ratings on 5-point Likert scales. The FAMSS was used to evaluate the core aspects of asthma management and was rated according to nine subscales, two of which included medication adherence and collaboration with healthcare provider. After the administration of the interview, dyads' responses were rated on a scale from 1=poor management to $9=$ excellent management using a standard manual that provides instructions and detailed rating guidelines for each subscale (McQuaid et al., 2005). The information considered in this subscale includes the "availability and use of quick relief medications and adherence to long-term controller medications" (McQuaid et al., 2005). Patient-provider collaboration was measured according to the FAMSS rating score for collaborative relationship with provider. Items for consideration in this subscale include "relationship with identified care provider, including effective 
communication, agreement regarding treatment approach; provider follows established guidelines for management, provides action plan” (McQuaid et al., 2005).

The objective assessment of adherence was based on baseline data. At the baseline study visit, participants were asked which ICS (i.e. Advair, Flovent, Symbicort, etc.) they had been prescribed and were using for the past 12 months. The pharmacy refill data was obtained over the phone. The participants' pharmacies reported the number of times the participant refilled any of their ICS prescriptions over the 12 month period prior to enrollment in the study. This provided a baseline measurement to be used in the calculation of the participant medication possession ratios (MPR), a common method of objectively measuring medication adherence. In this study, MPR was defined as the number of times a participant refilled their ICS prescription over a 12-month period. Prescriptions for asthma controller medications are typically filled in 30-day (one month) supplies (Sherman, Hutson, Baumstein, Hendeles, 2000). This information was used to calculate the MPR by dividing the number of times the prescription was filled by the observed time period (12 months) (Hess, Raebel, Conner \& Malone, 2006). An ideal MPR would be $12 / 12$, where the participant refilled their ICS prescription 12 times within the 12-month period.

All data used in this study was collected at baseline. The pharmacy refill data and the FAMSS scores for both adherence to asthma medications and collaborative relationship with provider were analyzed using the Statistical Package for the Social Sciences (SPSS). Pearson bivariate correlations were conducted to examine associations between both measures of asthma medication adherence and patient-provider collaboration. The basic descriptive information for this group of participants was also obtained from SPSS.

\section{Results}

The study involved participation from 30 adolescent-caregiver dyads. The adolescents in the study ranged in age from 12-15 and all of them had poorly-controlled asthma at the time of screening. The average age of the participants was 13.20 years. Of the 30 adolescents, 17 were male and 13 were female. The majority of the adolescents identified as African American $(n=19)$, and the mean total household income for the past year was $\$ 25,541.70$ (see Table 1). 
Table 1. Participant Demographic Variables

\begin{tabular}{lcc}
\hline & n & \% \\
\hline Gender & & 54.8 \\
\hline Male & 17 & 41.9 \\
Female & 13 & \\
\hline Ethnicity & 4 & 12.9 \\
\hline Caucasian/White & 19 & 61.3 \\
African American & 3 & 9.7 \\
Hispanic/Latino & 3 & 9.7 \\
Multi-racial & 1 & 3.2 \\
Asian & & \\
\hline Income & 5 & 16.1 \\
\hline \$500-\$6,999 & 4 & 12.9 \\
$\$ 8,000-\$ 13,999$ & 7 & 22.6 \\
$\$ 18,000-\$ 34,999$ & 5 & 16.1 \\
$\$ 35,000-\$ 100,000+$ & 3 & 9.7 \\
Don't know & $\boldsymbol{M} \pm$ SD & Range \\
\hline Age & $13.2 \pm 1.21$ & $12-15$ \\
\hline
\end{tabular}

The first aim examined the association between the subjective measure of asthma medication adherence and patient-provider collaboration. Pearson correlation coefficients revealed a positive association between FAMSS medication adherence scores and FAMSS collaborative relationship with provider $(r=.53, p=.003)$. A higher rating of medication adherence was associated with better patient-provider collaboration. The second aim evaluated the relationship between the objective measure of asthma medication adherence and patient-provider collaboration. The correlation between MPR and the FAMSS collaborative relationship with provider subscale did not demonstrate a significant relationship $(r=.27, p=.176)$. The correlation between the two measures of adherence was also non-significant $(r=.35, p=.068)$ (see Table 2$)$.

Table 2. Pearson Bivariate Correlations of Primary Study Variables

\begin{tabular}{|c|c|c|c|}
\hline Variable & 1 & 2 & 3 \\
\hline (1) Pharmacy Refill (MPR) & - & -- & -- \\
\hline FAMSS Adherence to Asthma Medications & .35 & -- & -- \\
\hline FAMSS Collaborative Relationship with Provider & .27 & $.53 *$ & -- \\
\hline
\end{tabular}




\section{Discussion}

It was hypothesized that both the objective and subjective measures of medication adherence would be positively associated with patient-provider collaboration. This hypothesis was partially supported in that the subjective measure of adherence using the FAMSS data was significantly associated with patient-provider collaboration. This suggests that higher medication adherence may be associated with more patient-provider collaboration. However, the objective measure of adherence via pharmacy refill data was not significantly related to patient-provider relationship. This may be attributed to the idea that pharmacy refill data does not imply that patients are actually taking their medication; it only measures medication possession (Lehmann et al. 2014). Interestingly, the individual measures of adherence were not correlated with each other. Due to small sample size, there may not have been enough power to detect an effect. On the other hand, this could indicate that the measures assess adherence differently. The FAMSS data incorporates use of participant self-report and researcher ratings, while MPR is solely based on pharmacy refill data.

The findings of this study support previous literature indicating that better medication adherence may be linked to a stronger patient-provider relationship (Sleath et al., 2012). The information in this study emphasizes the importance of the role the provider plays in the transition of care that occurs in adolescence. A treatment plan that both the patient and the provider understand and agree upon is ideal in ensuring treatment adherence. Results of this study show that a more collaborative plan may be more likely to be adhered to, potentially increasing the low rates of adherence observed in the studied age group. In addition, the multimethod assessment of adherence used in this study supports literature indicating objective assessments are needed to quantify adherence and that the combination of subjective and objective measures is the most useful (Krishnan et al., 2012).

The current study had several limitations, which may have affected the outcomes of the subjective and objective measures of adherence. One limitation in this study was the use of subjective measurements in the FAMSS interview. On average, the FAMSS interview was approximately 43 minutes long which may have induced fatigue in the participants. In addition, the use of self-report and researcher ratings in the FAMSS may have led to response bias. Another limitation in this study was the small sample size and the restricted location of data collection. This inhibits the generalizability of the results obtained in the study. There were also 
some limitations that resulted due to reliance on pharmacy refill data. Some participants may have obtained sample medications from their provider's office, thus eliminating the need for a refill from their pharmacy. Information about the previous use of inhalers was not considered, which may have resulted in discrepancies for participants who used multiple inhalers within the observed time period. In addition, the participants' pharmacy refill data may have been impacted if they switched from one pharmacy to another during the study. Since information on only one pharmacy was received, many pharmacies did not have a complete history of ICS refills.

The findings of this study emphasize the importance the patient-provider relationship has in patient medication adherence and disease self-management. However, it is still unclear which methods are the most appropriate for measuring medication adherence in adolescents with poorly controlled asthma. Future studies should examine data for a broader and larger sample from multiple sites. Additional studies should also assess adherence using various subjective and objective methods since combined approaches have been found to be most effective (Krishnan et al., 2012). The studies should consider using electronic device monitors for inhalers, obtaining refill information directly from the insurance companies, and incorporating information from multiple informants (i.e., physicians, families, and patients). Use of a multi-method approach to adherence assessment could increase the impact these studies have on asthma morbidity in adolescents.

\section{References}

Akinbami, L. J., Simon, A. E., \& Rossen, L. M. (2015). Changing trends in asthma prevalence among children. Pediatrics, peds-2015.

Bauman, L. J., Wright, E., Leickly, F. E., Crain, E., Kruszon-Moran, D., Wade, S. L., \& Visness, C. M. (2002). Relationship of adherence to pediatric asthma morbidity among inner-city children. Pediatrics, 110(1), e6-e6.

Brown, M. T., \& Bussell, J. K. (2011, April). Medication adherence: WHO cares? In Mayo Clinic Proceedings (Vol. 86, No. 4, pp. 304-314). Elsevier.

Bruzzese, J. M., Bonner, S., Vincent, E. J., Sheares, B. J., Mellins, R. B., Levison, M. J., ... \& Evans, D. (2004). Asthma education: the adolescent experience. Patient Education and Counseling, 55(3), 396-406.

Drotar, D., \& Bonner, M. S. (2009). Influences on adherence to pediatric asthma treatment: a review of correlates and predictors. Journal of Developmental \& Behavioral Pediatrics, 30(6), 574-582. 
Fedele, D. A., McConville, A., Thomas, J. G., McQuaid, E. L., Janicke, D. M., Turner, E. M., ... \& AbuHasan, M. (2018). Applying Interactive Mobile health to Asthma Care in Teens (AIM2ACT): Development and design of a randomized controlled trial. Contemporary Clinical Trials, 64, 230237.

Hess, L. M., Raebel, M. A., Conner, D. A., \& Malone, D. C. (2006). Measurement of adherence in pharmacy administrative databases: a proposal for standard definitions and preferred measures. Annals of Pharmacotherapy, 40(7-8), 1280-1288.

Jones, M. A. (2008). Asthma self-management patient education. Respiratory Care, 53(6), 778-786.

Krishnan, J. A., Bender, B. G., Wamboldt, F. S., Szefler, S. J., Adkinson, N. F., Zeiger, R. S., ... \& Adherence Ancillary Study Group. (2012). Adherence to inhaled corticosteroids: an ancillary study of the Childhood Asthma Management Program clinical trial. Journal of Allergy and Clinical Immunology, 129(1), 112-118.

Lehmann, A., Aslani, P., Ahmed, R., Celio, J., Gauchet, A., Bedouch, P., ... \& Schneider, M. P. (2014). Assessing medication adherence: options to consider. International Journal of Clinical Pharmacy, 36(1), 55-69.

Liu, A. H., Zeiger, R., Sorkness, C., Mahr, T., Ostrom, N., Burgess, S., ... \& Manjunath, R. (2007). Development and cross-sectional validation of the Childhood Asthma Control Test. Journal of Allergy and Clinical Immunology, 119(4), 817-825.

McQuaid, E. L., Kopel, S. J., Klein, R. B., \& Fritz, G. K. (2003). Medication adherence in pediatric asthma: reasoning, responsibility, and behavior. Journal of Pediatric Psychology, 28(5), 323-333.

McQuaid, E. L., Walders, N., Kopel, S. J., Fritz, G. K., \& Klinnert, M. D. (2005). Pediatric asthma management in the family context: The family asthma management system scale. Journal of Pediatric Psychology, 30(6), 492-502.

Modi, A. C., \& Quittner, A. L. (2006). Barriers to treatment adherence for children with cystic fibrosis and asthma: what gets in the way? Journal of Pediatric Psychology, 31(8), 846-858.

Morton, R. W., Everard, M. L., \& Elphick, H. E. (2014). Adherence in childhood asthma: the elephant in the room. Archives of Disease in Childhood, archdischild-2014.

National Asthma Education and Prevention Program (NAEPP) Expert Panel Report 3 (EPR-3): Guidelines for the Diagnosis and Management of Asthma-Summary Report 2007. Journal of Allergy and Clinical Immunology, 2007;120: S94-138.

Randolph, C., \& Fraser, B. (1999). Stressors and concerns in teen asthma. Current Problems in Pediatrics, 29(3), 82-93.

Rhee, H., Belyea, M. J., Ciurzynski, S., \& Brasch, J. (2009). Barriers to asthma self-management in adolescents: Relationships to psychosocial factors. Pediatric Pulmonology, 44(2), 183-191.

Sherman, J., Hutson, A., Baumstein, S., \& Hendeles, L. (2000). Telephoning the patient's pharmacy to assess adherence with asthma medications by measuring refill rate for prescriptions. Journal of Pediatrics, 136(4), 532-536.

Sleath, B., Carpenter, D. M., Slota, C., Williams, D., Tudor, G., Yeatts, K., ... \& Ayala, G. X. (2012). Communication during pediatric asthma visits and self-reported asthma medication adherence. Pediatrics, 130(4), 627-633. 
Taylor, D. R., Bateman, E. D., Boulet, L. P., Boushey, H. A., Busse, W. W., Casale, T. B., ... \& Kerstjens, H. A. M. (2008). A new perspective on concepts of asthma severity and control. European Respiratory Journal, 32(3), 545-554.

Walders, N., Drotar, D., \& Kercsmar, C. (2000). The allocation of family responsibility for asthma management tasks in African-American adolescents. Journal of Asthma, 37(1), 89-99.

Walders, N., Kopel, S. J., Koinis-Mitchell, D., \& McQuaid, E. L. (2005). Patterns of quick-relief and long-term controller medication use in pediatric asthma. Journal of pediatrics, 146(2), 177-182.

Zahran, H. S., Bailey, C. M., Qin, X., \& Moorman, J. E. (2014). Assessing asthma severity among children and adults with current asthma. Journal of Asthma, 51(6), 610-617. 